PROCEEDINGS OF THE

AMERICAN MATHEMATICAL SOCIETY

Volume 136, Number 12, December 2008, Pages 4461-4466

S 0002-9939(08)09567-1

Article electronically published on July 1, 2008

\title{
ON COMPACTIFICATIONS WITH PATH CONNECTED REMAINDERS
}

\author{
JAN J. DIJKSTRA
}

(Communicated by Alexander N. Dranishnikov)

\begin{abstract}
We prove that every separable and metrizable space admits a metrizable compactification with a remainder that is both path connected and locally path connected. This result answers a question of P. Simon.
\end{abstract}

Connectedness and compactness are two fundamental topological properties. A natural question is whether a given space admits a connected (Hausdorff) compactification. This question has been studied extensively; see [1, 5, 7, 9, 11. We mention both a positive and a negative result. It was proved by Alas, Tkačenko, Tkachuk, and Wilson 1 that a separable and metrizable space admits a metrizable and connected compactification provided the space contains no proper subsets that are open and compact. Confirming a conjecture of van Douwen 4, Emeryk and Kulpa 5] proved that the famed Sorgenfrey line does not admit a connected compactification.

We focus on the question whether a given space $X$ admits a compactification $C$ such that the remainder $C \backslash X$ is connected. Our research was prompted by a question asked by Simon [10, namely whether the space one obtains when one removes a countable dense subset from the unit square $[0,1]^{2}$ has some compactification such that the remainder is connected. Simon conjectured that the answer should be no. We present a general result that in particular shows that the answer to Simon's question is yes.

Theorem 1. Every separable and metrizable space admits a metrizable compactification with a remainder that is both path connected and locally path connected.

Proof. Let $X$ be a separable and metrizable space. Select a metrizable compactification $C$ of $X$ and let $d$ be a metric on $C$ that generates the topology. Select a countable dense subset $A$ in the remainder $C \backslash X$. If $C \backslash X$ is finite, then $X$ is locally compact and we can use the one-point compactification, so we may assume that $A$ is infinite. Let $\left\{a_{i}: i \in \mathbb{N}\right\}$ enumerate $A$ in such a way that $a_{i} \neq a_{j}$ if $i \neq j$. The idea of the proof is as follows. We first construct a new compactification $D$ of $X$ from $C$ by "blowing up" the points $a_{n}$ to intervals $I_{n}$. We then "glue" the $I_{n}$ together, producing a quotient space $E$ of $D$ that also compactifies $X$ and in which the image of $\bigcup_{n=1}^{\infty} I_{n}$ is a path connected set that is dense in the remainder.

Received by the editors October 30, 2007.

2000 Mathematics Subject Classification. Primary 54D40, 54D05.

Key words and phrases. Separable metric space, compactification, remainder, path connected, locally path connected.

(C)2008 American Mathematical Society Reverts to public domain 28 years from publication 
Moreover, by doing the gluing carefully we can arrange that the remainder is locally path connected.

Select for each $n \in \mathbb{N}$ a sequence of distinct points $x_{n}^{1}, x_{n}^{2}, \ldots$ in $X$ such that $\lim _{i \rightarrow \infty} x_{n}^{i}=a_{n}$. Let $\left\{q_{i}: i \in \mathbb{N}\right\}$ enumerate the rational numbers in the interval $[0,1]$. Since the set $\left\{x_{n}^{i}: i \in \mathbb{N}\right\}$ is discrete and closed in $C \backslash\left\{a_{n}\right\}$, we can extend any function from this set into $[0,1]$ to a continuous function on $C \backslash\left\{a_{n}\right\}$ by the Tietze Extension Theorem. In particular, we can find for each $n \in \mathbb{N}$ a continuous function $f_{n}: C \backslash\left\{a_{n}\right\} \rightarrow[0,1]$ such that $f\left(x_{n}^{i}\right)=q_{i}$ for each $i \in \mathbb{N}$. We define the function $f: C \backslash A \rightarrow[0,1]$ by

$$
f=\sum_{n=1}^{\infty} 2^{-n} f_{n}
$$

and we note that $f$ is continuous by the Weierstraß M-test. We now consider the graph $G$ of $f$ as a subspace of the product $C \times[0,1]$. Let $D$ denote the compactum that is the closure of $G$ in $C \times[0,1]$ and let $\pi: D \rightarrow C$ be the projection mapping. We define the metric $\rho((x, r),(y, s))=d(x, y)+|r-s|$ on $C \times[0,1]$ and we put $U(a, \varepsilon)=\{b \in D: \rho(a, b)<\varepsilon\}$ for $a \in D$ and $\varepsilon>0$. Since $f$ is continuous the natural mapping from $C \backslash A$ to $G$ is a homeomorphism and $G$ is a closed subset of $(C \backslash A) \times[0,1]$. Consequently, we have that $D$ is a compactification of the copy $X^{\prime}=\{(x, f(x)): x \in X\}$ of $X$, and for every $x$ in $C \backslash A$ we have

$$
\pi^{-1}(x)=D \cap(\{x\} \times[0,1])=\{(x, f(x))\} .
$$

For every natural number $n$ consider the continuous function $g_{n}:(C \backslash A) \cup\left\{a_{n}\right\} \rightarrow$ $[0,1]$ that is defined by

$$
g_{n}=\sum_{i=1}^{n-1} 2^{-i} f_{i}+\sum_{i=n+1}^{\infty} 2^{-i} f_{i} .
$$

Thus $f=g_{n}+2^{-n} f_{n}$ on $C \backslash A$. Put $I_{n}=\pi^{-1}\left(a_{n}\right)=D \cap\left(\left\{a_{n}\right\} \times[0,1]\right)$ for $n \in \mathbb{N}$.

Claim 2. For every $n \in \mathbb{N}$ we have

$$
I_{n}=\left\{a_{n}\right\} \times\left[g_{n}\left(a_{n}\right), g_{n}\left(a_{n}\right)+2^{-n}\right] .
$$

Proof. Note that on $C \backslash A, g_{n} \leq f \leq g_{n}+2^{-n}$ and hence by the continuity of $g_{n}$ in $a_{n}$ we have $I_{n} \subset\left\{a_{n}\right\} \times\left[g_{n}\left(a_{n}\right), g_{n}\left(a_{n}\right)+2^{-n}\right]$. Now let $t \in\left[0,2^{-n}\right]$. Select a subsequence $\left(q_{m(i)}\right)_{i}$ of $\left(q_{j}\right)_{j}$ such that $\lim _{i \rightarrow \infty} q_{m(i)}=2^{n} t$. Then $\lim _{i \rightarrow \infty} x_{n}^{m(i)}=$ $a_{n}$ and

$$
\begin{aligned}
\lim _{i \rightarrow \infty} f\left(x_{n}^{m(i)}\right)=\lim _{i \rightarrow \infty}\left(g_{n}\left(x_{n}^{m(i)}\right)+2^{-n}\right. & \left.f_{n}\left(x_{n}^{m(i)}\right)\right) \\
& =g_{n}\left(a_{n}\right)+2^{-n} \lim _{i \rightarrow \infty} q_{m(i)}=g_{n}\left(a_{n}\right)+t .
\end{aligned}
$$

Consequently, we have that $\left(a_{n}, g_{n}\left(a_{n}\right)+t\right)$ is in the closure $D$ of $G$. The claim is proved.

If $n, k \in \mathbb{N}$, we let $\mathrm{J}_{n}^{k}$ be the regular cover of the interval $I_{n}$ by $2^{k}$ closed intervals of length $2^{-n-k}$, that is,

$$
\mathcal{J}_{n}^{k}=\left\{\left\{a_{n}\right\} \times\left[g_{n}\left(a_{n}\right)+(i-1) 2^{-n-k}, g_{n}\left(a_{n}\right)+i 2^{-n-k}\right]: 1 \leq i \leq 2^{k}\right\} .
$$

Claim 3. The set $T=\bigcup_{i=1}^{\infty} I_{n}$ is dense in $D \backslash X^{\prime}$. 
Proof. Let $x \in C \backslash(X \cup A)$ and let $U$ be an open neighbourhood of $(x, f(x))$ in $D$. By the compactness of $D$ and the fact that $\pi^{-1}(x)=\{(x, f(x))\}$ we have that $V=C \backslash \pi(D \backslash U)$ is an open neighbourhood of $x$. Select an $a_{k}$ in $V$ and note that $I_{k}$ is contained in $U$. The claim is proved.

If $\varepsilon>0$, then a subset $N$ of a metric space is called an $\varepsilon$-net for that space if every point of the space has distance less than $\varepsilon$ towards $N$. Since $\rho$ is a metric on a compact space we can find an $\varepsilon$-net for $T$ for each $\varepsilon>0$. Thus we can construct a sequence $n(2)<n(3)<\cdots$ of natural a numbers such that $\bigcup_{j=1}^{n(i)} I_{j}$ is a $2^{-i}$-net for $T$ for each $i \geq 2$. Put $n(1)=1$. For all $J, K \in \bigcup_{i=1}^{n(2)} \mathcal{J}_{i}^{2}$, we choose points $y_{J K} \in J$ and $z_{J K} \in K$ and we define the collection

$$
\mathcal{P}_{2}=\left\{\left\{y_{J K}, z_{J K}\right\}: J, K \in \bigcup_{i=1}^{n(2)} \mathcal{J}_{i}^{2}\right\} .
$$

Since $\bigcup_{i=1}^{n(2)} \mathcal{J}_{i}^{2}$ is finite and we are choosing points in nondegenerate intervals, we can arrange that all the points are distinct so that $\mathcal{P}_{2}$ is pairwise disjoint.

It is easily seen that by recursion we can find collections $\mathcal{P}_{2} \subset \mathcal{P}_{3} \subset \mathcal{P}_{4} \subset \cdots$ of subsets of $T$ such that for $m \geq 2$,

(1) $\mathcal{P}_{m}$ is pairwise disjoint and finite;

(2) every element of $\mathcal{P}_{m}$ consists of two points;

(3) if $J, K \in \bigcup_{i=1}^{n(m)} \mathcal{J}_{i}^{m}$ and $\rho(J, K)<2^{-m+3}$, then there is a $P \in \mathcal{P}_{m} \backslash \mathcal{P}_{m-1}$ that intersects both $J$ and $K$; and

(4) if $m>2$, then every element of $\mathcal{P}_{m} \backslash \mathcal{P}_{m-1}$ has diameter less than $2^{-m+3}$.

We put $\mathcal{P}=\bigcup_{n=2}^{\infty} \mathcal{P}_{n}$. A sequence of subsets $B_{1}, B_{2}, \ldots$ of a metric space is called a null sequence if $\lim _{n \rightarrow \infty} \operatorname{diam} B_{n}=0$. It follows immediately from property (4) and the finiteness of the $\mathcal{P}_{n}$ that the elements of $\mathcal{P}$ can be arranged to form a null sequence. Consider now the decomposition

$$
\mathcal{D}=\mathcal{P} \cup\{\{x\}: x \in D \backslash \bigcup \mathcal{P}\}
$$

of $D$ and let $E$ be the corresponding quotient space with quotient map $q: D \rightarrow E$. Since $\mathcal{P}$ forms a null sequence of pairwise disjoint closed subsets of a compact space, we have by [8, Corollary A.11.6] that $E$ is a compact metrizable space. Note that $q \uparrow X^{\prime}: X^{\prime} \rightarrow q\left(X^{\prime}\right)$ is a homeomorphism and that $X^{\prime \prime}=q\left(X^{\prime}\right)$ is dense in $E$; thus $E$ is a metrizable compactification of a copy of $X$.

Claim 4. The set $q(T)$ is path connected.

Proof. Let $i \geq 2$ and let $m$ be such that $n(m-1)<i \leq n(m)$. If $m=2$, then by the definition of $\mathcal{P}_{2}$ the set $q\left(I_{i}\right)$ has a point in common with $q\left(I_{1}\right)$. If $m>2$, then $\bigcup_{j=1}^{n(m-1)} I_{j}$ is a $2^{-m+1}$-net; thus there is a $j \leq n(m-1)<i$ with $\rho\left(I_{i}, I_{j}\right)<2^{-m+1}$. Then by property (3) of $\mathcal{P}$ the sets $q\left(I_{i}\right)$ and $q\left(I_{j}\right)$ intersect. Thus the sequence $q\left(I_{1}\right), q\left(I_{2}\right), \ldots$ consists of path connected subsets of the remainder $E \backslash X^{\prime \prime}$ such that for each $i \geq 2$, the set $q\left(I_{i}\right)$ has a point in common with one of the preceding sets. According to [3, Theorem 5.14] we have that $q(T)$ is path connected.

From Claim 3 and the continuity of $q$ we may conclude that $q(T)$ is dense in $E \backslash X^{\prime \prime}$. Since the closure of a connected set is connected we have that $E \backslash X^{\prime \prime}$ is connected.

Claim 5. If $q(T) \subset Y \subset \overline{E \backslash X^{\prime \prime}}$, then $Y$ is path connected and locally path connected. 
Proof. Since $q(T)$ is dense in $Y$ and path connected it suffices to prove that $Y$ is locally path connected. Let $c$ be an arbitrary point in $Y$ and let $U$ be a neighbourhood of $c$ in $E$. We will show that every point $b \in q^{-1}(c)$ has a neighbourhood $V \subset q^{-1}(U)$ such that $Y \cap q(V)$ is contained in the path component $P_{c}$ of $c$ in $Y \cap U$. Since $q$ is a closed map it follows that $P_{c}$ is a neighbourhood of $c$ in $Y$, and we may conclude that $Y$ is locally connected.

Let $b \in D$ be such that $q(b)=c$. If $b \notin \bar{T}$, then since $q^{-1}(q(b))=\{b\}$ and $q$ is closed we have $q(b) \notin q(\bar{T})=\overline{E \backslash X^{\prime \prime}}$, a contradiction. So we have that $b \in \bar{T}$. Select a $k \in \mathbb{N}$ such that $U\left(b, 2^{-k+2}\right)$ is contained in $q^{-1}(U)$. Let $m(1)$ be the first index such that $\rho\left(b, I_{m(1)}\right)<2^{-k+1}$ and select a $J_{1} \in J_{m(1)}^{k-1}$ such that $\rho\left(b, J_{1}\right)<2^{-k+1}$. Since diam $J_{1}=2^{-m(1)-k+1} \leq 2^{-k}$ we have $J_{1} \subset U\left(b, 2^{-k+2}\right) \subset q^{-1}(U)$.

Let $x \in q^{-1}(Y)$ be an arbitrary element of $V=U\left(b, 2^{-k}\right)$. Note that $\rho\left(x, J_{1}\right)<$ $2^{-k+2}$. For each $i \geq 2$ let $m(i)$ be the first index such that $\rho\left(x, I_{m(i)}\right)<2^{-k-i+2}$. We obviously have $m(2) \leq m(3) \leq \cdots$ and $m(i) \leq n(k+i-2)$ for $i \geq 2$. Moreover, since $\rho\left(b, I_{m(2)}\right)<2^{-k+1}$ we have $m(1) \leq m(2)$. For every $i \geq 2$ select a $J_{i} \in \mathrm{J}_{m(i)}^{k+i-2}$ such that $\rho\left(x, J_{i}\right)<2^{-k-i+3}$. Note that for $i=1$ the same inequality holds. Thus for $i \in \mathbb{N}$ we have

$$
\rho\left(J_{i}, J_{i+1}\right) \leq \rho\left(x, J_{i}\right)+\rho\left(x, J_{i+1}\right)<2^{-k-i+3}+2^{-i-k+2}<2^{-i-k+4} .
$$

Since $J_{i}$ is a union of two elements of $\mathrm{J}_{m(i)}^{k+i-1}$ and $J_{i+1} \in \mathrm{J}_{m(i+1)}^{k+i-1}$, we have by property (3) of $\mathcal{P}$ for $m=k+i-1$ and the fact $m(i) \leq m(i+1) \leq n(k+i-1)$ that there exist a $y_{i} \in J_{i}$ and a $z_{i} \in J_{i+1}$ such that $q\left(y_{i}\right)=q\left(z_{i}\right)$.

For every $i \geq 1$ let $p_{i}:\left[\frac{1}{i+1}, \frac{1}{i}\right] \rightarrow J_{i+1}$ be a continuous map such that $p_{i}\left(\frac{1}{i}\right)=z_{i}$ and $p_{i}\left(\frac{1}{i+1}\right)=y_{i+1}$. We define $p:[0,1] \rightarrow Y$ by $p(0)=q(x)$ and $p\left\lceil\left[\frac{1}{i+1}, \frac{1}{i}\right]=q \circ p_{i}\right.$ for $i \in \mathbb{N}$. Since $q\left(y_{i}\right)=q\left(z_{i}\right)$ the function $p$ is well defined and continuous on $(0,1]$. We have for $i \geq 2$, diam $J_{i}=2^{-m(i)-k-i+2} \leq 2^{-k-i+1}<2^{-k}$ and $\rho\left(x, J_{i}\right)<$ $2^{-k-i+3} \leq 2^{-k+1} ;$ thus

$$
J_{i} \subset U\left(x, 2^{-k-i+4}\right) \cap U\left(b, 2^{-k+2}\right) \subset q^{-1}(U) .
$$

This means that $\lim _{i \rightarrow \infty} J_{i}=x$, and hence by the continuity of $q$ we have $\lim _{i \rightarrow \infty} q\left(J_{i}\right)=q(x)$. Thus $\lim _{i \rightarrow \infty} p\left(\left[\frac{1}{i+1}, \frac{1}{i}\right]\right)=q(x)$ and $p$ is also continuous in 0 . We have that $p((0,1]) \subset \bigcup_{i=2}^{\infty} q\left(J_{i}\right) \subset U$. Since $p(1)=q\left(z_{1}\right)=q\left(y_{1}\right) \in q\left(J_{1}\right) \subset U$ we have for every $x \in V \cap q^{-1}(Y)$ a path $p$ in $Y \cap U$ that connects $q(x)$ with the path connected subset $q\left(J_{1}\right)$ of $U$. This means that the path component of $q(b)$ in $Y \cap U$ includes $Y \cap q(V)$. Claim 5 is proved.

The theorem is proved.

In view of Claim 5 we have the following result.

Corollary 6. Every separable metrizable space has a metrizable compactification such that the closure of the remainder is a Peano continuum.

A space is called nowhere compact if every compactum in the space has an empty interior. Since compactifications of nowhere compact spaces have dense remainders we have:

Corollary 7. Every nowhere compact separable metrizable space has a metrizable compactification that is a Peano continuum. 
Remark 8. A better result than Corollary 7 follows immediately from a theorem of Bowers [2]. Let $Q=[0,1]^{\mathbb{N}}$ be the Hilbert cube and let $s=(0,1)^{\mathbb{N}}$ be its pseudointerior. Bowers proved that every nowhere compact separable metrizable space admits a dense imbedding in $s$. Consequently, such spaces have a compactification that is a Hilbert cube. Moreover, the remainder contains the pseudo-boundary of $Q$ and hence it is an absolute retract; see [8, Theorem 4.1.6 and Corollary 5.4.11].

Question 9. Does every separable and metrizable space admit a metrizable compactification with an absolute retract as remainder?

Remark 10. It is evident that if a space $X$ has a compactification $C$ with a connected remainder, then $C$ is connected if and only if $X$ contains no proper subsets that are compact and open. Thus the theorem of Alas et al. that we quoted at the beginning of this note follows immediately from our theorem. Observe also that nonempty open subsets of the Sorgenfrey line are not compact; thus the theorem of Emeryk and Kulpa implies that every compactification of that space has a disconnected remainder.

Remark 11. One easily constructs Tychonoff spaces with the property that they admit connected compactifications but no connected remainders. Let $X$ be a Tychonoff space such that its Cech-Stone remainder $\beta X \backslash X$ is infinite (for instance, a space that is not locally compact). We may select a countable infinite subset $A$ of $\beta X \backslash X$ that is not compact (if $A$ is discrete, then it is not compact; and if $A$ is not discrete, then we simply remove a limit point from $A$ ). Consider the space $Y=\beta X \backslash A$ and note that $\beta Y=\beta X$ by [6. Corollary 3.6.7]. Let $C$ be a compactification of $Y$. There exists a continuous surjection $f: \beta X \rightarrow C$ such that $f^{-1}(C \backslash Y)=A$; see [6, Theorem 3.5.7]. Thus we have that $C \backslash Y$ is countable and infinite because if $C \backslash Y$ were finite, then $A$ would have to be compact. We may conclude that $C \backslash Y$ is disconnected by [6. Corollary 6.1.3]. Thus if $X$ is connected we have that every compactification of $Y$ is connected but no remainder is connected.

Gruenhage, Kulesza, and Le Donne [7] proved that every nowhere compact metrizable space has a connected Hausdorff compactification.

Question 12. Does every metrizable space admit a Hausdorff compactification with a connected remainder?

If the answer is yes, then every metrizable space without compact and open proper subsets has a connected Hausdorff compactification, answering a question in [1].

\section{ACKNOWLEDGMENT}

The author thanks Jan van Mill for observations that led to Remarks 8 and 11 .

\section{REFERENCES}

[1] O. T. Alas, M. G. Tkačenko, V. V. Tkachuk, and R. G. Wilson, Connectifying some spaces, Topology Appl. 71 (1996), 203-215. MR.1397942 (98g:54039)

[2] P. L. Bowers, Dense embeddings of nowhere locally compact separable metric spaces, Topology Appl. 26 (1987), 1-12. MR893799 (88g:54029)

[3] F. H. Croom, Principles of Topology, Saunders, Philadelphia, 1989.

[4] E. K. van Douwen, Remote Points, Dissertationes Math. 188 (1981), 1-45. MR627526 (83i:54024) 
[5] A. Emeryk and W. Kulpa, The Sorgenfrey line has no connected compactification, Comment. Math. Univ. Carolinae 18 (1977), 483-487. MR0461437 (57:1422)

[6] R. Engelking, General Topology, PWN, Warsaw, 1975. MR0500779 (58:18316a)

[7] G. Gruenhage, J. Kulesza, and A. Le Donne, Connectifications of metrizable spaces, Topology Appl. 82 (1998), 171-179. MR1602455 (99a:54014)

[8] J. van Mill, The Infinite-Dimensional Topology of Function Spaces, North-Holland, Amsterdam, 2001. MR 1851014 (2002h:57031)

[9] J. R. Porter and R. G. Woods, Subspaces of connected spaces, Topology Appl. 68 (1996), 113-131. MR1374077 (97a:54020)

[10] P. Simon, Two questions about connected compactifications, Topology Atlas (2003), http://at.yorku.ca/q/a/a/a/22.htm.

[11] S. Watson and R. G. Wilson, Embeddings in connected spaces, Houston J. Math. 19 (1993), 469-481. MR:1242433 (94k:54040)

Faculteit der Exacte Wetenschappen/Afdeling Wiskunde, Vrije Universiteit, De Boelelaan 1081A, $1081 \mathrm{HV}$ Amsterdam, The Netherlands

E-mail address: dijkstra@cs.vu.nl 